\title{
Personalentwicklung ist Maßarbeit
}

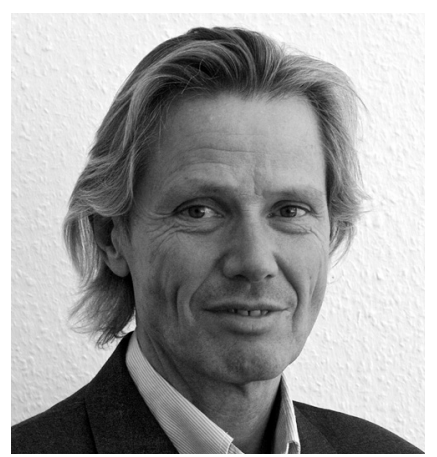

VON HANS-PETER KRÖSKE Hans-Peter Kröske ist Leiter der Personalentwicklung des Internationalen Bunds (IB) in Frankfurt am Main. Zuvor war er Vice President für Personal der CEMA AG und Trainer und Berater bei Wiebel \& Partner. Der Internationale Bund beschäftigt als Verein und mit seinen Gesellschaften mehr als 11.000 Mitarbeiterinnen und Mitarbeiter in 700 Einrichtungen an 300 Orten in Deutschland.

\author{
Strategische Personalentwicklung ist vor allem \\ Persönlichkeitsentwicklung. Diesen Grundsatz befolgt \\ der Internationale Bund (IB) als einer der großen \\ Anbieter der Jugend-, Sozial- und Bildungsarbeit.
}

Der Arbeitsmarkt im sozialen Bereich in Deutschland wird sich in den nächsten Jahren in der Folge des demografischen Wandels drastisch verändern. Es wird eine noch größere Nachfrage nach Gesundheits- und Pflegeleistungen geben, denn bis 2020 werden weitere rund fünf Millionen Menschen älter als 65 Jahre sein. Gleichzeitig werden in Deutschland insgesamt weniger Menschen leben. Der Rückgang wird besonders stark bei der Gruppe der unter Zwanzigjährigen ausfallen. Ihre Zahl wird bis zum Jahr 2020 um zwei Millionen sinken. Allein diese beiden skizzierten Entwicklungen zeigen, vor welchen Herausforderungen die Personalgewinnung und die Personalentwicklung von Unternehmen wie dem Internationalen Bund (IB) stehen.

Um als Personalentwickler dem Unternehmen wirklich zu geeigneten Mitarbeiterinnen und Mitarbeitern verhelfen zu können, müssen die Angebote maßgeschneidert zu den jeweiligen Lebens- und Arbeitssituationen passen. Mitarbeiterinnen und Mitarbeiter müssen sich bewusst sein oder werden, was sie brauchen, um in ihrem Arbeitsumfeld engagiert und selbstverantwortlich arbeiten zu können. Auch dabei können wir sie unterstützen.

Doch wie kann das dauerhaft gelingen? Wichtig ist dabei der Umgang mit und die Haltung gegenüber den Mitarbeiterinnen und Mitarbeitern. Unsere drei Grundprinzipien sind dabei: Aufmerksamkeit, Offenheit und Verbindlichkeit - und zwar vom ersten Kontakt an bis möglicherweise zum letzten, wenn es beispielsweise um eine Trennung geht. Ermöglicht wird dieser persönliche, indi- viduelle und von Verlässlichkeit geprägte Umgang bei den jeweils benötigten Lösungen vor allem durch eine professionelle Organisation.

\section{Vereinbarkeit von Familie und Beruf wird immer wichtiger}

Wir achten unter anderem darauf, dass unsere Mitarbeiterinnen und Mitarbeiter ihre Arbeit für den IB immer besser mit ihren privaten Bedürfnissen und Interessen in Einklang bringen können. Unser Ziel ist es, das Spektrum unserer Angebote so zu organisieren, dass sie für jede Phase in ihrem (Arbeits-) Leben hilfreich sind.

Das fängt mit Einführungstagen für neue Mitarbeiterinnen und Mitarbeiter an. Sie werden in den jeweiligen Organisationseinheiten angeboten. Wichtige Führungspersönlichkeiten stellen sich vor, die neuen Kolleginnen und Kollegen bekommen einen Überblick über unser Unternehmen. Diese Einführungstage sind eine gute Gelegenheit, die neuen Mitarbeiterinnen und Mitarbeiter spüren zu lassen, dass wir auf sie und ihre Kompetenzen setzen und sie uns herzlich willkommen im Team sind.

Für neue Führungskräfte wurde darüber hinaus ein Mentoring-Programm entwickelt. Das erleichtert ihnen den Start, hilft ihnen, sich gezielt zu vernetzen und die Besonderheiten der Unternehmenskultur ihres neuen Arbeitgebers kennenzulernen.

»Betroffene zu Beteiligte machen « - dieses Prinzip erleben die Mitarbeiterinnen und Mitarbeiter von Anfang an. Sie sind eingeladen, vor Ort oder 


\section{personal und recht \\ Produktportfolio}

\section{Personalentwicklung}
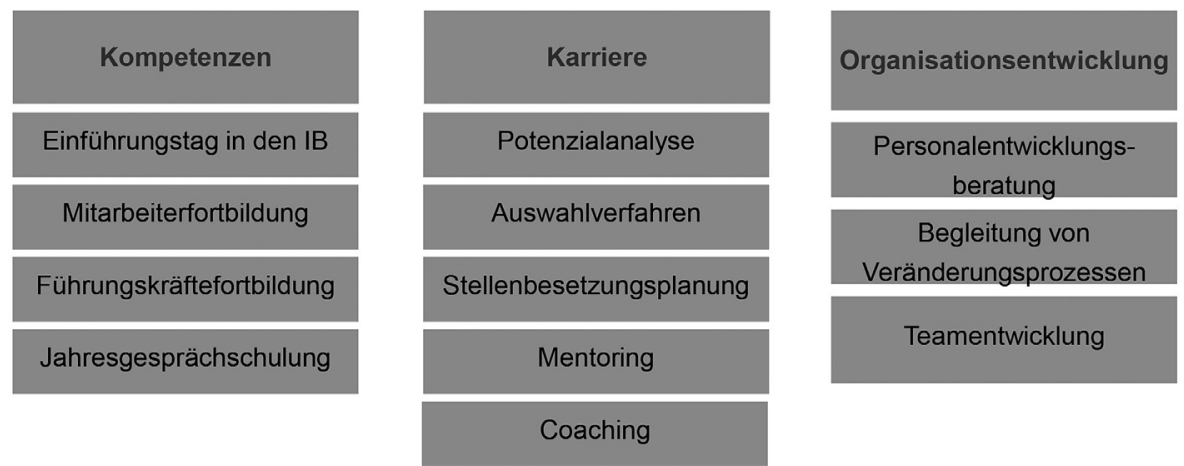

Der Internationale Bund (IB) hat für seine Dienste und Einrichtungen eine Reihe von Instrumenten der Personalentwicklung konzipiert, die es Mitarbeitenden ermöglichen sollen, ihre Arbeit für das Unternehmen mit ihrer privaten Lebenslage in Einklang bringen zu können.

IB-weit in Workshops oder bei Konferenzen mitzuarbeiten, um beispielsweise Geschäftsfelder weiterzuentwickeln oder bestehende Prozesse auf ihre Wirkung und Funktionalität hin zu überprüfen.

\section{Persönlichen Entwicklungsbedarf identifizieren}

Das IB-Jahresgespräch hat sich seit seiner Einführung vor mehr als zehn Jahren als wesentliches Führungsinstrument erwiesen. Damit diese Gespräche professionell geführt werden können, sind inzwischen alle Führungskräfte entsprechend geschult worden. Der zielgerichtete und in gewisser Weise formalisierte Dialog ermöglicht es, den ganz persönlichen Entwicklungsbedarf zu identifizieren, Karrierewünsche aufzunehmen und entsprechende Vereinbarungen treffen.

Mit speziellen Assessment-Centern für IB-Mitarbeiterinnen und Mitarbeiter, die Verantwortung innerhalb des Unternehmens übernehmen möchten, haben wir einen systematischen Einstieg in die Karriereentwicklung geschaffen. In den dreitägigen Veranstaltungen bieten wir Kolleginnen und Kollegen ein persönliches Potenzial-Feedback und skizzieren einen individuellen Unterstützungsplan. Seit 2010 bestehen die Grundvoraussetzungen für eine Fachlaufbahn, daher können die
Empfehlungen auch diesen Karrierepfad betreffen. Durch den kombinierten Einsatz von externen Moderatoren und geschulten internen Führungspersonen stoßen diese Empfehlungen bei den interessierten Mitarbeiterinnen und Mitarbeitern erfahrungsgemäß auf hohe Akzeptanz.

Ein bemerkenswertes Ergebnis der erfolgreichen Arbeit mit den Nachwuchsführungskräften ist ein daraus entstandenes Netzwerk. Die Personalentwicklung stärkt den Zusammenhalt dieser Gruppe durch die so genannte »NetzWERKstatt«. Einmal im Jahr dient sie als Plattform für den IB-weiten Austausch des Führungsnachwuchses untereinander.

\section{Jährliche Bedarfserhebung für die Mitarbeiterfortbildung}

Basis unseres Fort- und Weiterbildungsangebots ist eine jährliche Bedarfserhebung. Durch die Einbindung aller Mitarbeiterinnen und Mitarbeiter bei dieser Erhebung stellen wir sicher, dass die Trainings und Seminare den persönlichen und unternehmerischen Bedürfnissen und Notwendigkeiten entsprechen. Das Angebot reicht von der Rubrik »Arbeit mit Kindern und Jugendlichen « bis hin zu Modulen für das Führungstraining. In Zusammenarbeit mit dem Betriebsrat entsteht daraus der Fortbildungskatalog »Kompetenz und Karriere», der auch über eine eigens ge-

\section{"Die drei Grundprinzipien der Personalarbeit sind Aufmerksamkeit, Offenheit, Verbindlichkeit - und zwar vom ersten Kontakt an«}

Um nächste Karriereschritte strukturiert zu ermöglichen, haben wir weitere Verfahren wie das Entwicklungs-AC oder den Development-Prozess für die Ebene der Geschäftsführungen etabliert. staltete Webseite abgerufen werden kann (http://ib-personalentwicklung.de).

Seit einigen Jahren überprüfen wir die Wirkung der Trainings- und Seminare nicht nur durch einen Feedbackbogen 


\section{Neue Struktur der Führungs-Seminare im IB}

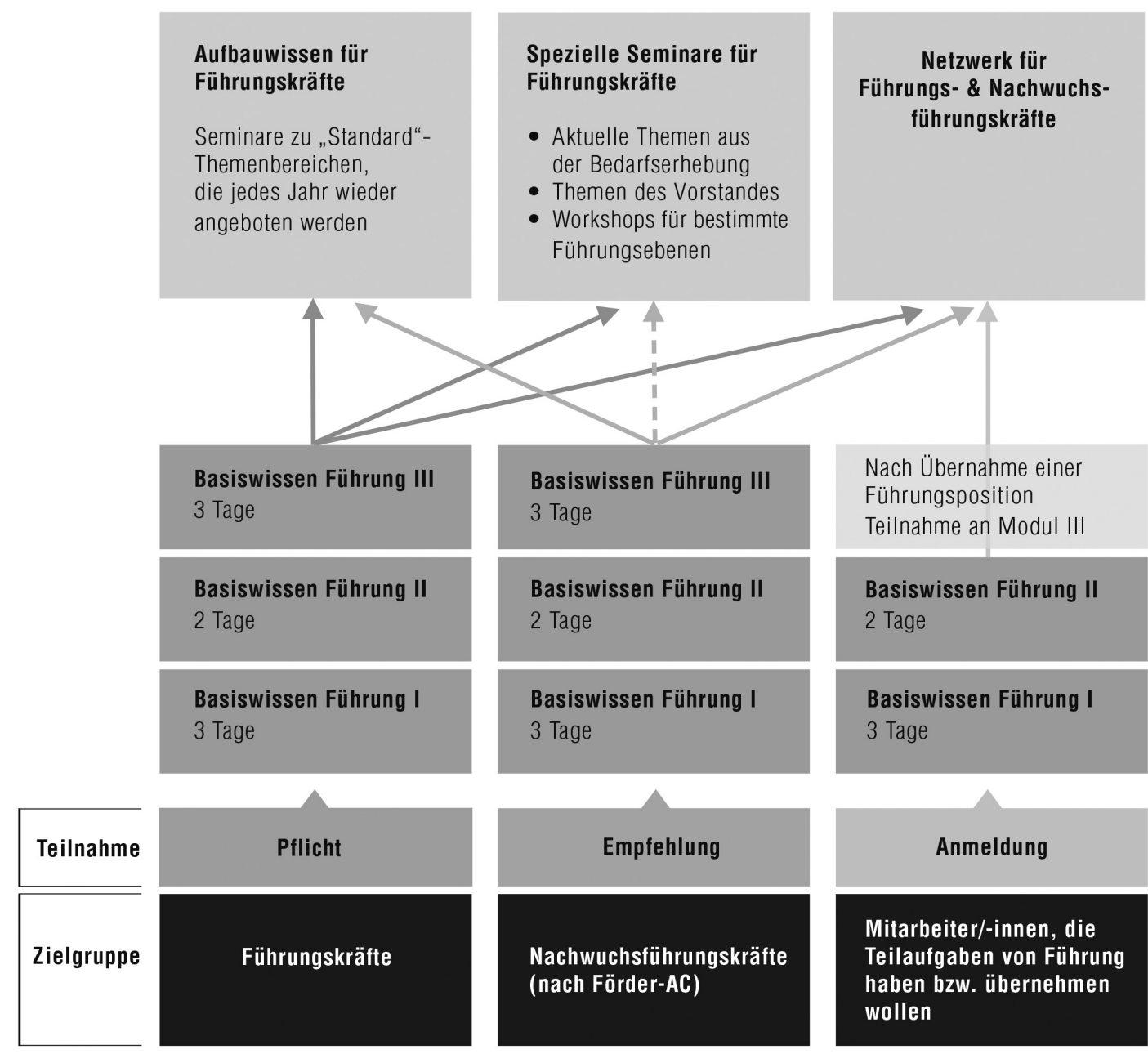

Für neue und etablierte Führungskräfte hat der Internationale Bund (IB) verschiedene Seminarreihen entwickelt. Ergänzt wird dieses Konzept durch eine »NetzWERKstatt«, die den Zusammenhalt der Führungskräfte stärken kann.

am Ende der Veranstaltung. Wir nehmen ein ausgewähltes Transfercontrolling vor, um die Wirkung im Arbeitsalltag zu überprüfen und die Fortbildungsangebote gegebenenfalls praxisorientiert verbessern zu können.

Das Gehaltsniveau in unserer Branche ist eher niedrig, bei gleichzeitigen hohen fachlichen und persönlichen Herausforderungen für die Mitarbeiterinnen und Mitarbeiter. Um neue Fachkräfte gewinnen und erfahrene Kolleginnen und Kollegen halten zu können, haben wir in den vergangenen Jahren neue Wege beschritten. Wir achten besonders auf die Vereinbarkeit von Beruf und Familie, bieten beispielsweise Feriencamps für Kinder von Mitarbeiterinnen und Mitarbeitern und ermöglichen sehr persönlich zugeschnittene Arbeitszeitmodelle.

So wurde nach anfänglich großer Skepsis auch das Modell »Führen in
Teilzeit« beim IB akzeptiert. Es wird inzwischen immer häufiger in Anspruch genommen.

\section{Unternehmen müssen attraktive Arbeitgeber sein}

Zusammengefasst haben wir alle Bemühungen in diesem Bereich unter dem Dach einer Arbeitsgruppe mit dem Namen »Der IB als attraktiver Arbeitgeber «. Hier werden kontinuierlich entsprechende neue Angebote für unsere Mitarbeiterinnen und Mitarbeiter entwickelt. Im Mittelpunkt stehen dabei vor allem die Mitarbeiterinnen und Mitarbeiter, die bereits für den IB tätig sind. Wir sind der Ansicht, dass es besser ist, sie im Unternehmen zu halten, als neue suchen zu müssen.

Natürlich vernachlässigen wir dennoch nicht das professionelle Personal- recruiting. In Deutschland bleibt im Schnitt eine Stelle 85 Tage lang unbesetzt, bis eine geeignete Nachfolgerin oder ein Nachfolger gefunden wird. Wir wollen nicht so lange warten müssen und arbeiten seit zwei Jahren daran, diese Vakanzzeit zu verkürzen. Dabei arbeiten die Mitarbeiter der Personalentwicklung und die für das Anwerben neuer Kolleginnen und Kollegen zuständigen Mitarbeiter eng und hierarchieübergreifend zusammen.

Unserer Erfahrung hängt der Erfolg der Personalentwicklung davon ab, wie wichtig ein Unternehmen die persönlichen Bedürfnisse der Mitarbeiterinnen und Mitarbeiter nimmt. Nur dann werden Arbeitgeber als attraktiv empfunden. Das wiederum kann den Ausschlag geben, wenn Bewerber sich zwischen mehreren Stellenangeboten entscheiden müssen. 